(C) 2015 IEEE. Personal use of this material is permitted. Permission from IEEE must be obtained for all other uses, in any current or future media, including reprinting/republishing this material for advertising or promotional purposes, creating new collective works, for resale or redistribution to servers or lists, or reuse of any copyrighted component of this work in other works. 


\section{A Modified Negative Selection Algorithm Applied in the Diagnosis of Voltage Disturbances in Distribution Electrical Systems}

\author{
Fernando P. A. Lima, Carlos R. Minussi \\ Electrical Engineering Department \\ UNESP, Univ. Estadual Paulista "Júlio de Mesquita Filho" \\ Ilha Solteira, SP, Brazil \\ engfernandoparra@gmail.com,minussi@dee.feis.unesp.br
}

\author{
Ricardo B. Bessa, José N. Fidalgo \\ Center of Power and Energy Systems (CPES) \\ INESC-TEC Porto \\ Porto, Portugal \\ ricardo.j.bessa@inescporto.pt, jfidalgo@inescporto.pt
}

\begin{abstract}
This paper presents a modified negative selection algorithm for the diagnosis of disturbance in distribution electrical systems. This study analyzes voltage disturbances and high-impedance faults, based on three phase current and voltage electric measures, which are obtained at the substations. The principal application is to support operation decision aid during faults, as well as to supervise the protection system. To evaluate the performance of the proposed method, simulations were executed using the EMTP software for a distribution test system containing 134 bus. The results obtained were compared with the specialized literature.
\end{abstract}

Index Terms-Electrical Distribution Systems, Voltage Disturbance, High-Impedance Fault, Modified Negative Selection Algorithm, Artificial Immune Systems.

\section{INTRODUCTION}

Nowadays the electrical power systems are continuously expanding due to the increasing of the population and industrial groups. Thus, the electrical energy providers began to invest in distributions systems to modernize and automate the operation and consequently increase the profits [1]. These investments seek satisfy the requirements of the regulator agencies, as well as to improve the reliability, security and the efficiency of the energy providing. Digital technology and information technology have been pointed out lately.

Therefore, it is necessary to develop integrated systems that combine several techniques, as acquisition, processing and analysis of data to aim providing the adequate assistance to provide automation, control and decision making.

The traditional methods of fault diagnosis are based on visual inspection of oscillation graphs and depend on the operator experience to analyze and decision making. It is emphasized that not all faults can be identified by the operators, and thus the process can be inefficient and unsecure. Therefore, the use of artificial intelligence is a plausible solution to the diagnosis problem. Several techniques have been used to aid the operators to execute routines providing security, velocity and efficiency in corrective planning actions.

According to this, some techniques are emphasized in the specialized literature in the last years for performing diagnosis of the disturbances in distribution electrical systems.

Reference [2] uses artificial neural networks. In [3] the methodology is fuzzy logic. Metha-heuristics as Genetic algorithm and Tabu Search are used in [4]. In [5], Petri nets are used. These works are some of the references as aid tools for decision and failure diagnosis in electrical distribution systems using artificial intelligence. However, the works more relevant to realize analysis and diagnosis of current and voltage electric disturbances in distribution systems are presented in the following paragraphs.

Reference [6] presents a study to evaluation of the low frequency spectrum in the diagnosis of high-impedance fault arcing on sandy soil in $15 \mathrm{kV}$ distribution feeders. Reference [7] proposes a voltage disturbance classification using wavelet and neural networks. In [8] the authors use neural networks, fuzzy logic and wavelet to voltage disturbance diagnosis. Reference [9] presents a methodology based on wavelet, entropy norm and multi layer perceptron (MLP) neural network trained by backpropagation to classify the voltage disturbance. This method estimates the detail coefficients approaching by wavelet, and these coefficients are aggregated by entropy norm. Thus, a MLP neural network trained by backpropagation is used to classify the disturbances.

A hybrid system based on discrete wavelet to detection process and a set of neural networks to classify the events is proposed in [10] realizing the detection, localization and

This work was supported in part by INESC-TEC Porto/Portugal, and in part by CAPES-PDSE/Brazil. 
classification of quality energy disturbances. The wavelet to extract the characteristics of the voltage oscilographs is used in [11], with a fuzzy ARTMAP neural network that classify the disturbances where the inputs are aggregated by entropy concept. Reference [12] presents an algorithm for detecting high-impedance faults using gradient multiresolution morphological in feature extraction in the time domain and multi-layer perceptron networks.

Paper [12] proposes a new approach to the problem to diagnosis of voltage disturbances using artificial immune systems. In this work, authors used the negative selection algorithm to perform the proper/non-proper discrimination. The proper signals indicate normal operation and the nonproper signals indicate where there is the presence of abnormality, which is classified by the algorithm. Reference [14] presents an abnormalities filter detector using artificial immune systems in Smart Grids systems for the storage occurrence of abnormalities in real electrical systems. This system is also effective for generating actual database for training, validation and testing of new intelligent methodologies.

This paper presents a new method to detect and classify voltage disturbances in distribution electrical system using an modified negative selection algorithm (MNSA). The modification in the negative selection algorithm was performed in the censor process (learning), which evaluates the similarity of the candidates for detectors. This evaluation computes the value of population combination (VPC), that will be used as a metric to choose the detectors in the censor process. The VPC is the degree of combination of the candidate with all other candidates for detectors. The bigger the candidate's VPC, the greater the power to identify and classify a similar pattern in the monitoring process.

This strategy provides greater efficiency in the learning process of the MNSA, and consequently, greater efficiency and accuracy in the diagnosis of disturbances.

To evaluate the performance this algorithm, tests were effectuated with two systems, one with 84 buses and a real system with 134 buses.

The present article is organized as follows. Section II presents MNSA. Section III describes the modelling and simulations. The section IV presents the methodology proposed. The following section presents some illustrative results. Section VI summarizes the main conclusions of this work.

\section{Modified Negative SElection Algorithm}

The MNSA algorithm proposed in this paper is an efficient technique for pattern recognition problem. The MNSA is based on the activity of the organic immunological system, and it was implemented as a computational model. The original technique was proposed in [15] for the detection of changes in computer systems, mimicking the negative selection process of $\mathrm{T}$ lymphocytes, responsible for discriminating between the body's own cells and foreign antigens.

This paper proposes a modification in the negative selection algorithm, in the censor process (learning), where an evaluation of similarity of the candidates for detectors is performed. The evaluation will calculate the value of population combination (VPC) that will be used as a metric to choose the detectors in the censor process. The bigger the candidate's VPC, the greater the power to identify and classify a similar pattern in the monitoring process. The MNSA algorithm is explained next:

1. Censor (Learning)

a) Define the proper chain set (S). This set represents the proper condition of the problem;

b) For each pattern type, perform the evaluation similarity process, obtaining the VPC for all detectors candidates for the pattern in analysis;

c) Generate a list with VPC of the candidates in decreasing order, where the maximum VPC is the first in the list;

d) Using the list, choose the candidates and evaluate the affinity (match) with each one and the proper chains. If the affinity is superior to a pre-defined value, the chain is rejected. Otherwise, it must be filed in a set of detectors (R);

\section{Monitoring}

a) Given a chain set that is desired to protect (protected chains), evaluate the affinity with each one and the detector set. If the affinity is superior to a pre-defined value, then the non-proper element is identified.

In the censor phase of MNSA the detectors are initially defined, which represent a normal condition of the body, known as proper chains (S). The objective of this phase is to generate a set of detector patterns (R), with the ability to recognize any non-proper pattern, in the data monitoring stage.

So, for each pattern type an analysis of the similarity is performed, where is obtained the VPC of each detector candidate. After this process, is created a list in decreasing order with the VPC of the candidates. The list is used for choose the candidates. This strategy provides efficient candidates, with major chance identification and classification in the monitoring process. In the original algorithm, this choice is performed randomly.

In the monitoring phase, the data is monitored in order to identify changes in the behavior of the samples and then classify these changes using the set of detectors created during the censor phase. Thus, by analyzing the protected chains (S) and comparing them with the set of detectors $(\mathrm{R})$ the affinity between each of the chains is evaluated. If the affinity exceeds a certain threshold, then the non-self element is detected and classified. It should be noted that the censor and monitoring phases are performed offline and in real-time, respectively.

It should be noted that the MNSA is very similar to the negative selection algorithm. However, it presents a much more efficient learning strategy, which allows greater precision in final diagnosis. 
The chains at the MNSA algorithm represent the patterns through the data structures (vectors). These chains can be classified in two types at the MNSA algorithm, the antigens (Ag) and the antibodies (Ab). The $\mathrm{Ag}$ is the signal to be analysed in the process and can be represented by expression (1). The detectors represent the antibodies $\mathrm{Ab}$ and are expressed according to expression (2) $[16,17]$ :

$$
\begin{aligned}
& A g=\left[A g_{1}, A g_{2}, A g_{3}, A g_{4}, \ldots, A g_{L}\right] \\
& A b=\left[A b_{1}, A b_{2}, A b_{3}, A b_{4}, \ldots, A b_{L}\right]
\end{aligned}
$$

where $L$ is the dimension of the space of the antigen and the antibody.

\section{A. Affinity and match criterium}

In order to evaluate the affinity between samples and to check his similarity, a match criterion is commonly used. Match may be considered perfect or partial. A perfect match occurs when both samples are identical. In the partial match case the similarity is compared to a predefined threshold. This threshold is designated as affinity rate, and represents the minimum correspondence accepted between two matched samples [18].

The approach developed in this work adopts the concept of partial match proposed in [18], where the affinity rate is defined according to the following equation:

$$
T A f=\left(\frac{A n}{A t}\right) * 100
$$

where:

TAf : affinity rate;

An : number of normal samples;

At : total number of samples.

Equation (3) allows the precise calculation of the affinity rate for the proposed problem and represents the statistical analysis of the samples of the problem. Expression (4) represents the method to quantify the total affinity with the analyzed patterns $[18,19]$ :

$$
A f_{T}=\frac{\sum_{i=1}^{L} P c_{i}}{L} * 100
$$

where:

$A f_{T} \quad$ : \% of affinity with the patterns analysed;

$L \quad$ : Total quantity of positions;

$P C_{i}$ : matched position;

$\sum_{i=1}^{L} P c_{i}:$ sum of the matched position.

Thus, if $A f_{T}$ is greater than $T A f$, the combination/matching with the patterns occurs, i.e., the patterns are considered equal (similar). Otherwise, there is no matching with the patterns.

\section{B. Similarity evaluation process}

The similarity evaluation process is performed to obtaining the VPC for the detectors candidates.

For this process, it is used the matching and affinity concept presented in the previous section, where an evaluation is performed with each pattern type, in order to obtain the VPC of the each candidate.
VPC represents the degree of candidate combination with all the other candidates, namely, the identification and classification capability. The VPC is defined according to the following equation:

$$
V P C=\frac{\sum_{i=1}^{N} M t h_{i}}{N} * 100
$$

where:

$V P C \quad: \%$ of similarity of the candidate with all candidates;

$N \quad$ : quantity of candidates analysed;

$M t h_{i} \quad$ : quantity of matching with all candidates;

$\sum_{i=1}^{N} M t h_{i}:$ sum of the matching.

The Mth value of each candidate is obtained by comparison, analysis and verification of the matching criterium and affinity, between the candidate and the other patterns. For each combination/matching, Mth is incremented. And so, calculate the value of VPC.

Thus, the higher the candidate's VPC, the greater its ability to identify and classify an abnormal pattern in the monitoring process.

\section{MODELING AND SIMULATIONS}

The electrical distribution systems are vulnerable to problems and failures during the operation. These failures can be produced by problems in equipments, grass burning, animal touches in energized line parts, natural phenomena. These events can cause interruptions in energy providing, or modify the energy quality introducing harmonics and lowering the quality indexes of the distribution companies, and then increase the operational costs.

Three principal disturbances can be emphasized in electrical distribution systems: short circuit, high-impedance fault and voltage disturbances. The short circuit and the highimpedance fault are related to electrical current problems, whereas the voltage disturbances, also known as power quality disturbances, are related to electrical voltage problems [14].

In this work are considered the voltage disturbances and the high-impedance fault. The voltage disturbances have the characteristics to present short time variations in voltage magnitude since a predefined nominal value. Depending on the magnitude variation, the disturbances are classified as interruptions, elevations and sinking, and depending on the duration can be instantaneous, transitory or temporary [11]. A high-impedance fault is characterized by the contact with an energized conductor and high-impedance surfaces, such as asphalt, sidewalks, and sand, or high-impedance objects, such as, branches and trees. These surfaces and objects limit the levels of the fault currents to values that are less than to those detected by the protection equipment [12].

The difficulties in effectuating researches in this area (distribution systems) are the data set under perturbations. Therefore, it is necessary to model the test systems, simulating events (voltage disturbances, short circuit, among others) 
providing data to use in the proposed method and contributing to the automation of the substations.

In this context, a real distribution system with 134 buses was modeled using the EMTP software [20]. The sample frequency used in the simulations was $15.36 \mathrm{kHz}$, which corresponds to 256 samples per cycle. The simulation time was $200 \mathrm{~ms}$, which corresponds to 12 cycles.

In total, 968 simulations were performed. The simulations consider the phase where the disturbance occurred, the loadings from $50 \%$ to $120 \%$ and the parameters of the theoretical model. Table I presents the simulation quantity for each type of disturbance simulated to evaluate the proposed methodology.

Table I - Quantify of simulations.

\begin{tabular}{cc}
\hline Disturbance & Number of simulations \\
\hline Normal operation & 200 \\
Voltage & 672 \\
High-impedance fault & 96 \\
\hline Total & 968 \\
\hline
\end{tabular}

The electrical system is simulated 968 times, where 200 simulations were done without perturbations and 768 simulations containing and at least some electrical disturbance (high impedance fault and voltage disturbance). To uniform the test set, 96 simulations were chosen for each kind of disturbance executed at the simulations, therefore, every disturbance has the same quantity of signals.

\section{A. $\quad$ Voltage disturbances}

The theoretical model proposed by [21] was used for the voltage disturbance simulations. In this theoretical model is presented equations and parameters to reproduce faults referring to outage, harmonic, swell, sag, swell with harmonic, sag with harmonic, oscillatory transient disturbances. This type of faults is introduced on EMTP simulator [20]. In this case, using the routine models [22] of the EMTP software [20], the sources were modelled for each type of voltage disturbances in the theoretical model.

A total of 672 voltage disturbance simulations were executed as shown in Table 2 .

Table II - Voltage disturbance simulations.

\begin{tabular}{cc}
\hline Disturbance type & Number of simulations \\
\hline Voltage Swell & 96 \\
Voltage Sag & 96 \\
Outage & 96 \\
Harmonics & 96 \\
Swell with Harmonics & 96 \\
Sag with Harmonics & 96 \\
Oscillatory transients & 96 \\
\hline Total & 672 \\
\hline
\end{tabular}

\section{B. High-impedance faults}

An approximated model proposed by [6] and shown in Figure 1 was used for the high-fault simulations. This model has two DC sources, which are connected by two diodes in anti-parallel manner. The magnitude of the current arch is controlled by the series impedance ( $\mathrm{R}$ and $\mathrm{X})$.
A total of 96 simulations were executed for the highimpedance fault while considering the variations of the parameters $R, X, V_{\mathrm{p}}$ and $V_{\mathrm{n}}$, and the location bus.

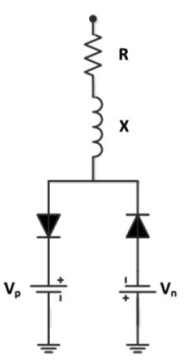

Figure 1: Model of High-impedance faults simulations.

\section{METHODOLOGY}

The diagnosis system based on the MNSA has two phases, the censor (learning) and monitoring, that are presented follows:

\section{A. Censor Phase}

The detectors are generated in this phase and the MNSA uses in the monitoring process. Figure 2 shows the flow chart of this process. This phase is executed offline and separated by phase, where each phase (three phase system) has the proper detectors.

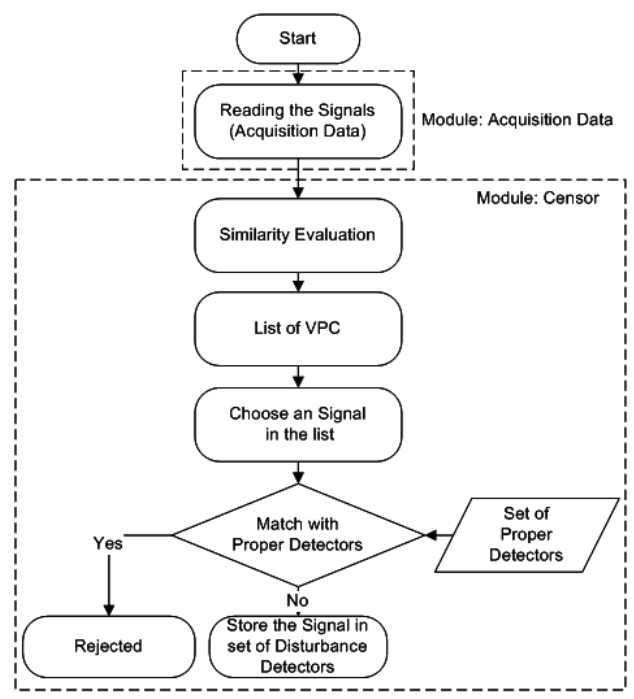

Figure 2: Flowchart of MNSA censor phase

Firstly the proper set is defined. The proper signals for the electrical distribution systems are the normal operation state. Then, window samples (256 points) of the signals representing the normal operation are filed as proper detectors. Afterwards, the censor module is executed to generate the disturbance detectors, emphasizing that this process is executed for each kind of disturbance. In this phase, the candidate detectors are evaluated for the obtaining the VPC, then, is generated a list in decreasing order. The list is used for choose the candidates. This process is repeated until a predefined quantity of detectors is obtained for each disturbance.

Thus, this process generates the detector patterns for each 
type of disturbance and files in the memory to realize the classification of the monitoring process.

\section{B. $\quad$ Monitoring phase}

The monitoring phase is divided in three modules, which realizes the data acquisition, the proper/non proper discrimination, and classifies the disturbances.

The data acquisition module reads the electrical current and voltage oscillographs measured at the substations for the three phases (by SCADA acquisition system). The sample rate used is $15.36 \mathrm{kHz}$, corresponding to 256 samples per cycle with $200 \mathrm{~ms}$, or 12 execution cycles, i.e. 12 windows.

Afterwards the detection proper/non proper module is executed. In this module the proper detectors and the signals under analysis are compared to identify a match according to the Affinity Rate. The generated diagnosis is a classification of the analyzed signals (proper and non-proper), being the proper signals considered the normal operation and the nonproper the identification of an anomaly. Figure 3 illustrates the proper/non proper detection process.

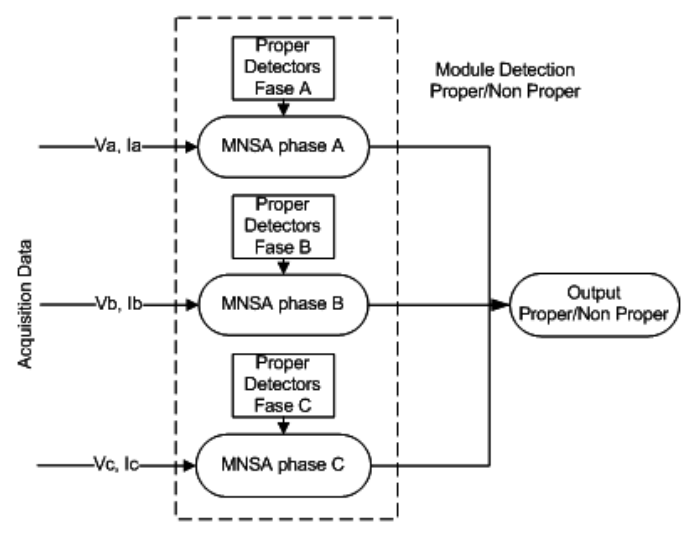

Figure 3: Module Detection proper/non proper.

For each phase of the system there is an artificial immunological system and the respective detectors. If some non-proper signal is identified, the system presents a codification how answer. A codification is used to represent the non-proper signal associated to a predefined phase of the system. Table III presents the codification for the output of the detection phase.

\begin{tabular}{cc} 
Table III - Proper/non proper output codification. \\
\hline Output & Codification \\
\hline Phase $A$ & 10 \\
Phase $B$ & 01 \\
Phase $C$ & 11 \\
\hline
\end{tabular}

An abnormality being detected, the proper/non-proper detection module actions the classification module to classify the disturbance detected in the phase where is identified a nonproper signal. The classification module identifies the kind of disturbance the anomaly is and classifies it among the disturbances known by the system. Figure 4 illustrates the classification module of the system.

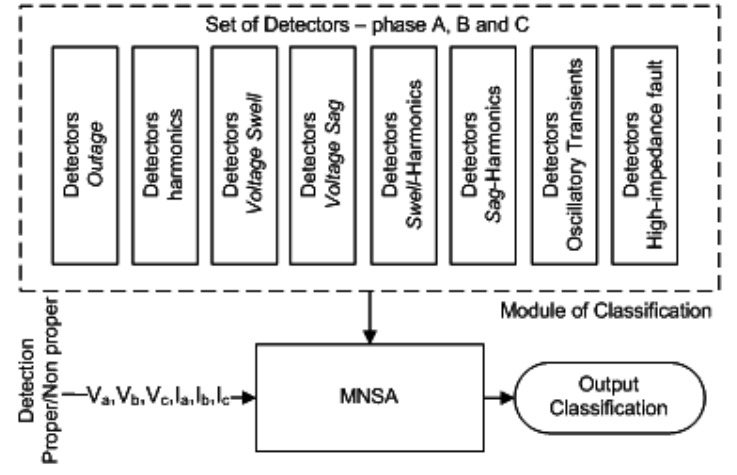

Figure 4: Module of Classification.

The execution of the module is similar to the proper/nonproper module. However, in this phase a comparison between the abnormal signal detected and the disturbances detectors is realized. In this work 8 disturbances are considered. Thus, in the final of this process the classification module gives a codified output for the 8 types of disturbances, i.e. the classification of the abnormal signal.

\section{RESULTS}

The results obtained for the proposed method for the electrical system is presented in this section. Every simulation is executed with a PC Intel Core 2 Duo $1.9 \mathrm{GHz}, 2 \mathrm{~GB}$ of RAM memory and operational system Windows 7 Ultimate 32 bits. The algorithm is developed in MATLAB [23].

\section{A. Real system of 134-Bus}

This system is a real distribution system that has the following characteristics: aerial, three phase, several branches, composed of 134-bus, $13.8 \mathrm{kV}, 7.065$ MVA, mutually coupled, and the power factor is equal to 0.92 [24]. This electrical system represents part of the distribution system of the hydroelectric power plant Jupiá in Três Lagoas city, São Paulo, Brazil.

The parameters used are the following: the affinity rate with a set value in $66.66 \%$ (calculated with equation (3)) and the number of the detector for each disturbance is $30 \%$ of the data. Table IV presents the results that were obtained with the MNSA for the 134-bus system [24].

Table IV - Results for the 134-Bus system.

\begin{tabular}{ccc}
\hline Disturbance & Tested Patterns & Match $(\%)$ \\
\hline Voltage Swell & 96 & 100.00 \\
Voltage Sag & 96 & 100.00 \\
Outage & 96 & 100.00 \\
Harmonics & 96 & 100.00 \\
Swell with Harmonics & 96 & 100.00 \\
Sag with Harmonics & 96 & 100.00 \\
Oscillatory transients & 96 & 100.00 \\
High-impedance fault & 96 & 100.00 \\
Normal operation & 200 & 100.00 \\
\hline Total & 968 & 100.00 \\
\hline
\end{tabular}

The results show the correct percentage in identifying the electrical current and voltage disturbances in relation to the quantity of patterns used on the tests. The monitoring phase is executed with a computational time less that to $100 \mathrm{~ms}$. 


\section{B. $\quad$ Comparative study}

Table $\mathrm{V}$ presents a comparative study to the proposed method and the principal references that are available in the literature.

\begin{tabular}{ccc}
\multicolumn{3}{c}{ Table V - Comparative study. } \\
\hline Reference & Type of Disturbance & $\begin{array}{c}\text { Total } \\
\text { Match (\%) }\end{array}$ \\
\hline$[9]$ & Voltage & 96.21 \\
{$[13]$} & Voltage & 99.11 \\
{$[10]$} & Voltage & 99.31 \\
{$[11]$} & Voltage & 99.66 \\
{$[6]$} & High-impedance fault & 97.85 \\
{$[12]$} & High-impedance fault & 99.20 \\
This work & Voltage & 100.00 \\
This work & High-impedance fault & 100.00 \\
\hline Total & & 100.00 \\
\hline
\end{tabular}

The proposed method presents a matching index and a precision that is superior to other similar works that are available in the literature. It should be emphasized that the MNSA proposed in this work, is an efficient technic, because the modification implemented provides a better learning in the censor phase, improving the accuracy in diagnosis.

\section{CONCLUSIONS}

This paper presents a new method for disturbance diagnosis in distribution electrical systems using the MNSA. The MNSA proposed presents good results with $100.00 \%$ matches in every sample simulated. The censor phase demands more computational time and thus it is executed offline. The monitoring phase is very fast (less that to $100 \mathrm{~ms}$ ), allowing the algorithm to be used on line, once the decisions must be executed very quickly. Therefore, the MNSA is efficient, robust and exact for the disturbance diagnosis in distribution electrical system.

\section{ACKNOWLEDGMENT}

This work is financed by the ERDF (European Regional Development Fund) through the COMPETE Programme (operational programme for competitiveness) and by National Funds through the FCT (Portuguese Foundation for Science and Technology) within project FCOMP-01-0124-FEDER037281. The authors also gratefully acknowledge CAPESPDSE (Proc. $N^{\circ}$ BEX 3659/14-3) by scholarship granted.

\section{REFERENCES}

[1] J. Northcote-Green; R. Wilson "Control and Automation of Electrical Power Distribution Systems," Taylor \& Francis, 2007.

[2] G. P. Amis; G. A. Carpenter. "Self-supervised ARTMAP Neural Networks," Oxford, v. 23, n. 2, pp. 265-282, 2010.

[3] L. A. Zadeh. "Fuzzy sets", Information and Control, New York, v. 8, n. 3, pp. 338-353. 1995.

[4] C. S. Chang; F. S. Wen. "Tabu search based approach to trouble call analysis." IEEE Gener. Transm. Distrib, vol. 145, No. 6, pp. 731-738. 1998.

[5] K. L. Lo; H. S. Ng; J. Trecat. "Power system fault diagnosis using Petri nets." IEEE Gener. Transm. Distrib., vol. 144, No. 3, pp. 231-236. 1997.
[6] A. E. Emanuel, E. M. Gulachenski, "High impedance fault arcing on sandy soil in $15 \mathrm{kV}$ distribution feeders: contributions to the evaluation of the low frequency spectrum." IEEE Transactions on Power Delivery, vol. 5, n. 2, pp. 676-686, 1990.

[7] S. Santoso; E. J. Powers; W. M. Grady; A. C. Parsons. "Power quality waveform recognition using wavelet-based neural classifier-Part 1: theoretical foundation", IEEE Transactions on Power Delivery, vol. 15, n. 1, pp. 222-228. 2000 .

[8] M. B. I. Reaz; F. Choong; M. S. Sulaiman; F. Mohd-Yasin; M. Kamada. "Expert system for power quality disturbance classifier." IEEE Transaction on Power Delivery, vol. 22, n. 3, 2007, pp. 1979-1988.

[9] M. Uyar; S. Yildririm; M. T. Gencoglu. "An effective wavelet-based feature extraction method classification of power quality disturbance signals." Electric Power System Research, vol. 78, n. 10, pp. 1747-1755. 2008.

[10] M. Oleskovicz; D. V. Coury; O. D. Felho, W. F. Usida; A. F. M. Carneiro; L. R. S. Pires "Power quality applying a hybrid methodology with wavelet transform and neural networks." International journal of electrical power \& Energy systems, vol. 31, n. 5, pp. 206-212, 2009.

[11] J.G.M.S. Decanini; M.S. Tonelli-Neto; F. C. V. Malange and C.R. Minussi "Detection and classification of voltage disturbances using a fuzzy-ARTMAP-wavelet network," Elec. Power Syst. Research, vol. 81, n. 12, pp. 2057-2065, 2011.

[12] M. Sarlak, S. M. Shahrtash, "High impedance fault detection using combination of multi-layer perceptron neural networks based on multiresolution morphological gradient features of current waveform." IET Generation, Transmission and Distribution, vol. 5, n. 5, pp. 588-595, 2011.

[13] F. P. A. Lima, A. D. P. Lotufo, C. R. Minussi, "Artificial immune systems applied to voltage disturbance diagnosis in distribution electrical systems," in Proc. 2013 IEEE PowerTech, pp. 1-6.

[14] F. P. A. Lima, A. D. P. Lotufo, C. R. Minussi, "Disturbance Detection for Optimal Database Storage in Electrical Distribution Systems Using Artificial Immune Systems with Negative Selection." Electric Power Systems Research, vol. 109, pp. 54-62, 2014.

[15] S. Forrest, A. Perelson, L. Allen, R. Cherukuri, "Self-nonself discrimination in a computer." in Proc. 1994 IEEE Symposium on Research in Security and Privacy, pp. 202-212.

[16] L. N. de Castro, Immune engineering: development and application of computational tools inspired by artificial immune systems, PhD. Thesis, UNICAMP, 2001. (in Portuguese).

[17] L. N. de Castro, J. Timmis, Artificial immune systems: a new computational intelligence approach, Springer-Verlag, vol. 1, 2002.

[18] D. W. Bradley, A. M. Tyrrell, "Immunotronics - novel finite-statemachine architectures with built-in self-test using self-nonself differentiation." IEEE Trans. Evolutionary Computation, vol. 6, pp. 227$238,2002$.

[19] F. P. A. Lima, A. D. P. Lotufo, C. R. Minussi, "Wavelet-artificial immune system algorithm applied to voltage disturbance diagnosis in electrical distribution systems." IET Generation, Transmission \& Distribution, pp. 1-8, 2015.

[20] EMTP-RV (Eletromagnetic Transient Program), Powersys-Solutions, Le Puy-Sainte-Réparade, France, 2011.

[21] T. K. Abdel-Galil; M. Kamel; A. M. Youssef; E. F. El-Saadany; M. M. A. Salama "Power quality disturbance classification using inductive inference approach," IEEE Transactions on Power Delivery, vol. 19, No. 4, pp. 1812-1818, 2004.

[22] L. Dubé, "How to use MODELS-based user-defined network components in ATP." Proc. of EEUG, Budapest, 1996, pp. 10-12.

[23] MATLAB: "7.8 Version", Mathworks Company, 2011.

[24] LaPSEE, 2011. Practical 134 bus feeder data. Available: http://www.dee.feis.unesp.br/lapsee/ Lines, Transactions of the AIEE, part III - Power Apparatus and Systems, v. 76 (Aug.), p. 518-539. 